\title{
Solenoidal versus compressive turbulence forcing
}

\author{
C. Federrath ${ }^{1}$, J. Duval ${ }^{2}$, R. S. Klessen ${ }^{1}$, W. Schmidt ${ }^{3}$ and \\ M.-M. Mac Low ${ }^{4}$ \\ ${ }^{1}$ Zentrum für Astronomie der Universität Heidelberg, Institut für Theoretische Astrophysik, \\ Albert-Ueberle-Str. 2, D-69120 Heidelberg, Germany \\ ${ }^{2}$ Astronomy Department at Boston University, \\ 725 Commonwealth Avenue, Boston, MA 02215, USA \\ ${ }^{3}$ Institut für Astrophysik Göttingen, \\ Friedrich-Hund-Platz 1, 37077 Göttingen, Germany \\ ${ }^{4}$ Department of Astrophysics, American Museum of Natural History, \\ Central Park West at 79th Street, New York, NY 10024-5192, USA
}

\begin{abstract}
We analyze the statistics and star formation rate obtained in high-resolution numerical experiments of forced supersonic turbulence, and compare with observations. We concentrate on a systematic comparison of solenoidal (divergence-free) and compressive (curl-free) forcing (Federrath et al. 2009 a,b), which are two limiting cases of turbulence driving. Our results show that for the same RMS Mach number, compressive forcing produces a three times larger standard deviation of the density probability distribution. When self-gravity is included in the models, the star formation rate is more than one order of magnitude higher for compressive forcing than for solenoidal forcing.
\end{abstract}

Keywords. hydrodynamics - ISM: clouds - ISM: structure - methods: statistical - turbulence

Observational data indicate that turbulence in the ISM exhibits both signatures of solenoidal and compressive forcing, depending on the region under consideration (Heyer et al. 2006; Hily-Blant et al. 2008; Goodman et al. 2009). In particular, expanding shells show statistical characteristics similar to compressively driven turbulence.

Compressive forcing produces a three times larger standard deviation of the threedimensional and the column density probability distributions (PDFs) for the same RMS Mach number (Federrath et al. 2008). When self-gravity is added to the models, the star formation rate is about 25 times higher for compressive forcing. These two results demonstrate that star formation models based on the turbulent density PDF (Padoan \& Nordlund 2002; Krumholz \& McKee 2005; Elmegreen 2008; Hennebelle \& Chabrier 2009) must take the nature of the turbulence forcing into account.

\section{References}

Elmegreen, B. G. 2008, ApJ, 672, 1006

Federrath, C., Duval, J., Klessen, R. S., Schmidt, W., \& Mac Low, M.-M. 2009a, arXiv:0905.1060

Federrath, C., Klessen, R. S., \& Schmidt, W. 2008, ApJ, 688, L79

Federrath, C., Klessen, R. S., \& Schmidt, W. 2009b, ApJ, 692, 364

Goodman, A. A., Pineda, J. E., \& Schnee, S. L. 2009, ApJ, 692, 91

Hennebelle, P. \& Chabrier, G. 2009, ApJ, 702, 1428

Heyer, M. H., Williams, J. P., \& Brunt, C. M. 2006, ApJ, 643, 956

Hily-Blant, P., Falgarone, E., \& Pety, J. 2008, A\&BA, 481, 367

Krumholz, M. R. \& McKee, C. F. 2005, ApJ, 630, 250

Padoan, P., \& Nordlund, A. 2002, ApJ, 576, 870 